\title{
EFFECT OF SESAMOL IN ASSOCIATION WITH FOLIC ACID ON 6-OHDA INDUCED PARKINSONIAN ANIMALS-BIOCHEMICAL, NEUROCHEMICAL AND HISTOPATHOLOGICAL EVIDENCE
}

\author{
KHADIRA SEREEN A ${ }^{1}$, VIJAYALAKSHMI $\mathrm{K}^{1 *}$, PRIYA NAGAPPAN ${ }^{1}$, SHINU BALIMA ${ }^{2}$ \\ ${ }^{1}$ Department of Biochemistry, Bharathi Women's College, Chennai, Tamil Nadu, India. ${ }^{2}$ Veterinarian, Microtherapeutics \\ Research Lab Pvt. Ltd., Padi, Chennai, Tamil Nadu, India. Email: viji42research@yahoo.co.in
}

Received: 20 May 2016, Revised and Accepted: 16 January 2017

\section{ABSTRACT}

Objective: Parkinson's disease (PD) is the world's second neurodegenerative disorder. Degeneration of dopaminergic neurons is the hallmark of the disease. Here is a novel approach to treat PD with a phenolic compound Sesamol (SA) and in combination with folic acid (FA).

Methods: The study was designed with five groups of animals and 6 rats in each group. The rats were infused with 6 -hydroxydopamine $(10 \mu \mathrm{g} / 2 \mu \mathrm{l}$ in $0.1 \%$ ascorbic acid saline) once for the development of PD, Group 1 (control), Group 2 (lesion), Group 3 (lesion+SA), Group 4 (lesion+SA+FA), and Group 5 (lesion+L-dopa). The biochemical parameters such as glucose, triglycerides, protein, FA, thiobarbituric acid reactive substance (TBARS), and antioxidant profile in serum were estimated. The neurotransmitters level in striatum was estimated, and histopathology of striatum and midbrain tissues was carried out.

Results: The results showed that 6-hydroxydopamine induced lesion has a significant alteration in the level of glucose, triglycerides, protein and FA, whereas TBARS level was elevated and the activities of antioxidants and neurotransmitters level were reduced. This was significantly restored on SA and FA treatment. The lesion group shows an abnormal architecture of striatum and midbrain, whereas on SA and FA treatment there was minimal abnormality.

Conclusion: Thus, our study demonstrates that SA has a neuroprotective effect against 6-hydroxydopamine insult and showed a synergic effect when combined with FA.

Keywords: Parkinson's disease, Sesamol, Folic acid, 6-hydroxydopamine, Neurotransmitter, Antioxidant.

(c) 2017 The Authors. Published by Innovare Academic Sciences Pvt Ltd. This is an open access article under the CC BY license (http://creativecommons. org/licenses/by/4. 0/) DOI: http://dx.doi.org/10.22159/ajpcr.2017.v10i4.12961

\section{INTRODUCTION}

Neurodegenerative disorders are common among elderly people. Parkinson's disease (PD) is the world's second neurodegenerative disorder next to Alzheimer's disease. The degeneration of dopaminergic neurons of substantia nigra pars compacta is the pathology of the disease. This kind of degeneration will reduce the level of neurotransmitter called dopamine (DA), which is involved in the locomotory event of the body. The DA depletion causes dysregulation in motor circuit of basal ganglia [1]. This leads to motor impairment such as bradykinesia, muscular rigidity, tremor, and postural instability. The degeneration of dopaminergic neurons may be due to oxidative stress, iron deposition, environmental toxins, mitochondrial dysfunction, and protein aggregation (Lewy body) [2]. The current medical strategy is just to alleviate the symptoms of the diseases. Although L-dopa was found to be the gold standard for the past 40 years, it causes side effects like sleep disturbance and emotional problems.

Oxidative stress is the basic precursor for almost all the deadly diseases and PD is also one among them [3]. Thus, it has been focused to find natural antioxidants such as curcumin, epigallocatechin-3-gallate, melatonin and so on to treat PD [4]. On this path, we have chosen the most powerful antioxidant sesamol (SA) present in sesame seeds. SA is a natural organic compound present in sesame oil extracted from Sesamum indicum that belongs to Pedaliaceae family. Numerous wild relative species are found in Africa and a smaller number in India. SA has various activities such as antioxidant, antifungal, neuroprotective [5], photoprotective [6], and hepatoprotective [7]. Along with SA, folic acid (FA) was combined, as FA involved in the synthesis of DA and it is to be noted that FA deficiency causes PD [8].
Therefore, this study was planned to evaluate the effect of SA in association with FA on 6-hydroxydopamine induced Parkinsonian animals in comparison with L-dopa. The general blood parameters such as glucose, triglycerides, protein, and FA were estimated. The activities of superoxide dismutase (SOD), catalase, glutathione peroxidase (GPx) and glutathione reductase (GRx), and the levels of glutathione (GSH), vitamin-C, vitamin-E and thiobarbituric acid reactive substance (TBARS) in serum of experimental animals were estimated. The levels of neurotransmitter such as DA, norepinephrine (NE), homovanillic acid (HVA), and 3,4-dihydroxy phenyl acetic acid (DOPAC) in striatum of animals were measured by flourimetric analysis. The tissue morphology of striatum and midbrain tissues was studied.

\section{METHODS}

\section{Chemicals}

6-hydroxydopamine, SA, L-dopa, apomorphine, FA, and ascorbic acid were purchased from Sigma-Aldrich. All the other chemicals used for the study were of analytical grade.

\section{Experimental protocol}

Male wistar rats of weight 200-250 g were purchased and maintained at $25 \pm 2^{\circ} \mathrm{C}$ in $12 \mathrm{hrs}$ light/dark cycle with free access to food and water. The animal protocol was approved by the Institutional Animal Ethical Committee of Saveetha University (SU/BRULAC/ $\mathrm{RD} / 004 / 2013)$. The rats were segregated into five groups, 6 rats in each group, Group 1 (control), Group 2 (lesion), Group 3 (lesion+SA), Group 4 (lesion+SA+FA), and Group 5 (lesion+L-dopa). The rats were anesthetized and infused with 6-hydroxydopamine at the 
rate of $1 \mu \mathrm{l} /$ minute, $2 \mu \mathrm{l}$ of 6-hydroxydopamine $(10 \mu \mathrm{g} / 2 \mu \mathrm{l}$ in $0.1 \%$ ascorbic acid) was injected intrastriatal in right striatum once for the development of PD. The regions were located according to rat brain atlas (antero-posterior $0.5 \mathrm{~mm}$, lateral $2.5 \mathrm{~mm}$, dorso-ventral $4.5 \mathrm{~mm}$ relative to bregma, and ventral from dura). After 21 days, the disease development was confirmed by Apomorphine induced contralateral rotational test [9]. The animals were treated with SA (30 mg/kg body weight, intrapritonially), FA ( $5 \mathrm{mg} / \mathrm{kg}$ body weight, orally), and L-dopa (100 mg/kg, orally) for the next 24 days.

\section{Sample collection}

After the experimental period ( 45 days), animals were sacrificed by cervical dislocation. Anesthesia was not given as it alters the brain amines [10]. Blood was collected by cardiac puncher. The brains are removed immediately, the striatum and midbrain was dissected and processed for neurochemicals and histopathological study.

\section{Biochemical parameters}

Estimation of blood glucose

The blood glucose level was estimated by 0-Toluidine method [11].

\section{Estimation of protein}

The protein was estimated by Lowry method [12].

\section{Estimation of triglycerides}

Triglycerides levels of experimental animals are estimated by the method of Rice [13].

\section{Estimation of FA}

FA in serum of experimental animals was estimated by the simple spectrophotometric method of Nagaraja et al. [14].

\section{Estimation of antioxidant profile in serum of animals}

Enzymatic antioxidants such as SOD [15], catalase [16], GPx [17] and GRx [18] and non-enzymatic antioxidant like GSH [19], vitamin-C [20] and vitamin-E [21]. Level of TBARS was also estimated as specified by Ohkawa et al. [22].

\section{Estimation of neurotransmitter levels in striatum of animals} DA and NE estimation

The striatum was homogenized with acidified butanol and centrifuged at $800 \mathrm{~g}$. Aliquots served as test samples. DA and NE were estimated by the method of Kari et al. [23]. The internal standard was prepared by known amount DA $(1 \mu \mathrm{g})$ and NE $(1 \mu \mathrm{g})$. The fluorescence of DA and NA was read at (385 and $485 \mathrm{~nm}$ ) and ( 320 and $370 \mathrm{~nm}$ ), respectively, with a slit wide of $10 / 10 \mathrm{~nm}$.

\section{HVA and DOPAC estimation}

The homogenized striatal tissue sample was extracted with tris for HVA estimation and 1,2-diaminoethen for DOPAC estimation according to method of Murphy et al. [24]. The fluorescence of HVA was measured at 315 and $430 \mathrm{~nm}$ and DOPAC was measured at 385 and $450 \mathrm{~nm}$, with $5 \mathrm{~mm}$ wide slit.

\section{Histopathological study}

The striatum and midbrain tissues were dissected from each group of animals. The tissues were fixed in $10 \%$ formalin and processed. The fixed tissues were embedded in paraffin wax and sectioned on the gelatine coated glass slide. The sections were stained with hematoxylin and eosin, then observed under light microscope. Brain tissue sections were examined for physiological changes and compared with the control group.

\section{Statistical analysis}

The results are expressed as mean \pm standard deviation one-wayanalysis of variance was applied to the data to derive the significance of the results. The $\mathrm{p}<0.001$ was considered statistically significant.

\section{RESULTS}

\section{Biochemical parameters}

Estimation of glucose, triglycerides, and protein (Table 1) shows that there is a significant variation $(\mathrm{p}<0.001)$ between control animals (Group 1) and lesion animals (Group 2), and this was restored by SA and FA treatment. In comparison with lesion animals SA and FA treated animals showed a significant increase in the level of glucose, triglycerides, and protein than SA treated animals and L-dopa treated animals. The FA level shown in Table 1 indicates that there is a significant difference between control animals and lesion animals. The SA and FA treated animals show normal level of FA, which was highly significant $\mathrm{p}<0.001$ when compared with lesion animals.

Enzymatic antioxidants activity in serum of experimental animals Table 2 indicates the enzymatic antioxidants activities of SOD, catalase, GRx and GPx. There was a significant decrease in the activity of these enzymes in the lesion group animals when compared with control group animals. But on treatment with SA, FA and L-dopa the enzyme activity has increased. Among three treatment groups, the SA and FA treated animals shows significant $(\mathrm{p}<0.001)$ increase in the activity when compared with lesion animals.

Table 1: Biochemical parameters in serum of experimental animals

\begin{tabular}{llllll}
\hline Parameters & Group 1 (control) & Group 2 (lesion) & Group 3 (lesion+SA) & Group 4 (lesion+SA+FA) & Group 5 (lesion+L-dopa) \\
\hline Glucose (mg/dl) & $111.89 \pm 6.19$ & $96.54 \pm 5.09^{*}$ & $98.87 \pm 2.62^{\mathrm{NS}}$ & $107.74 \pm 3.02^{\#}$ & $96.34 \pm 1.07^{\mathrm{NS}}$ \\
Triglycerides (mg/dl) & $74.46 \pm 1.17$ & $62.55 \pm 1.59^{*}$ & $68.29 \pm 2.61^{\#}$ & $71.09 \pm 1.54^{\#}$ & $64.30 \pm 1.27^{\mathrm{NS}}$ \\
Protein (g/dl) & $6.34 \pm 0.04$ & $6.05 \pm 0.03^{*}$ & $6.24 \pm 0.03^{\#}$ & $6.36 \pm 0.04^{\#}$ & $6.23 \pm 0.07^{\#}$ \\
FA (ng/mL) & $12.85 \pm 1.14$ & $10.25 \pm 0.37^{*}$ & $11.19 \pm 0.43^{\# \# \#}$ & $14.65 \pm 0.35^{\#}$ & $10.85 \pm 0.57^{\mathrm{NS}}$ \\
\hline
\end{tabular}

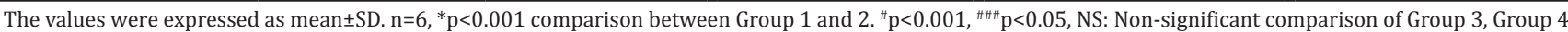
and Group 5 with Group 2. SA: Sesamol, FA: Folic acid, SD: Standard deviation

Table 2: Enzymatic antioxidants activity in serum of experimental animals

\begin{tabular}{|c|c|c|c|c|c|}
\hline Enzymes & $\begin{array}{l}\text { Group } 1 \\
\text { (control) }\end{array}$ & $\begin{array}{l}\text { Group } 2 \\
\text { (lesion) }\end{array}$ & $\begin{array}{l}\text { Group } 3 \\
(\text { lesion+SA) }\end{array}$ & $\begin{array}{l}\text { Group } 4 \\
(\text { lesion+SA+FA) }\end{array}$ & $\begin{array}{l}\text { Group } 5 \\
\text { (lesion+L-dopa) }\end{array}$ \\
\hline SOD U/mg protein & $5.41 \pm 0.15$ & $3.14 \pm 0.32 *$ & $3.69 \pm 0.16^{\#}$ & $4.75 \pm 0.26^{\#}$ & $3.41 \pm 0.16^{\# \#}$ \\
\hline Catalase $\left(\mu \mathrm{mol} \mathrm{H}_{2} \mathrm{O}_{2}\right.$ consumed/minute/mg protein) & $31.96 \pm 1.24$ & $21.37 \pm 0.59 *$ & $24.34 \pm 0.78^{\# \#}$ & $30.29 \pm 4.67^{\#}$ & $22.36 \pm 0.82^{\mathrm{NS}}$ \\
\hline GPx ( $\mu$ mol NADPH oxidize/minute/mg protein) & $3.65 \pm 0.10$ & $1.97 \pm 0.23^{*}$ & $2.38 \pm 0.14^{\#}$ & $3.20 \pm 0.17^{\#}$ & $2.94 \pm 0.12^{\#}$ \\
\hline GRx ( $\mu$ mol NADPH oxidize/minute/mg protein) & $2.90 \pm 0.21$ & $1.51 \pm 0.08^{*}$ & $1.81 \pm 0.10^{\# \#}$ & $2.52 \pm 0.13^{\#}$ & $2.10 \pm 0.11^{\#}$ \\
\hline
\end{tabular}

The values were expressed as mean \pm SD. $n=6,{ }^{*} p<0.001$ comparison between Group 1 and Group 2. ${ }^{p} p<0.001$, \#\#p<0.01, NS: Non-significant are comparison Group 3 , Group 4 and Group 5 with Group 2. SA: Sesamol; FA: Folic acid, SD: Standard deviation, SOD: Superoxide dismutase, GPx: Glutathione peroxidiase, GRx: Glutathione reductase, NADPH: Nicotinamide adenine dinucleotide phosphate 
Non-enzymatic antioxidants and TBARS level in serum of experimental animals

The levels of antioxidant such as GSH, vitamin-C, and vitamin-E of experimental animals were shown in Table 3. It was found that the antioxidant levels were significantly decreased in Lesion group animals when compared with control animals. This was restored by treatment. In comparison with Lesion group, Group 3, Group 4, and Group 5 animals show a significant increase $(\mathrm{p}<0.001)$ in levels. Group 4 animals show near normal level that is the SA and FA treated animals show a better effect than other groups. On the other hand, the level TBARS (Table 3) of Lesion group animals was significantly increased when compared with the control group. This was reduced significantly on SA and FA treatment.

\section{Neurotransmitter levels in striatum of experimental animals}

Fig. 1 shows the levels of DA, NE, DOPAC and HVA. It indicates the level of neurotransmitters in striatum of animals in Lesion group were significantly reduced $(\mathrm{p}<0.001)$ when compared with control group animals. On SA, FA, and L-dopa treatment the levels were increased. Among treatment groups, the SA and FA treated groups show increased levels of neurotransmitter which was highly significant $(\mathrm{p}<0.001)$ in comparison with lesion group.

Histopathological evidence of striatum and midbrain of experimental animals

Plate A1-A5 shows the histopathology of the striatum of experimental animals. The control animal (A1) shows the normal architecture of striatal tissue, whereas on 6-hydroxydopamine insult the changes such as cellular inflammation, vascular degeneration, and cytoplasmic vacuolation are visualized in lesion group animals (A2). There was an improvement on SA treated group (A3) and SA and FA treated group (A4), but there is no improvement in L-dopa treated group (A5). Among SA treated group and SA and FA treated group, the latter group shows better architecture of striatum similar to that of the control group.

Similarly, Plate B1-B5 showed the histology of midbrain of experimental animals. The control animals are with normal architecture, whereas the midbrain of lesion animals showed abnormalities like vascular

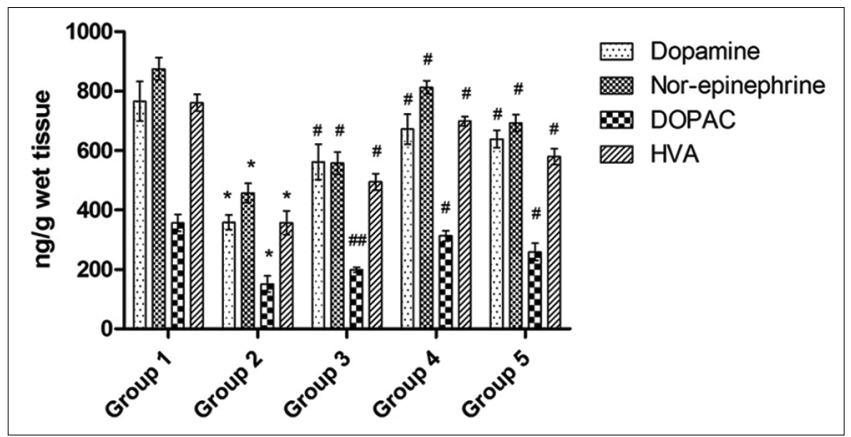

Fig. 1: Neurotransmitter levels in stratum of experimental animals. The values were expressed as mean \pm standard deviation. $\mathbf{n}=6 .{ }^{*} \mathbf{p}<0.001$ comparison between Groups 1 and $2 .{ }^{*} \mathbf{p}<0.001$ comparison of Group 3, Group 4, and Group 5 with Group 2. Group 1 - Control, Group 2 - Lession, Group 3 - Sesamol treated, Group 4 Sesamol and folic acid treated, and Group 5 - L-dopa treated degeneration and vaculation. These abnormalities are at minimal on SA treatment, and Group 4 showed less degeneration than Group 3. The L-dopa treated animals showed abnormality as in the case of lesion animals. The histopathological study evidenced that the degeneration of neuron in striatum and midbrain caused due to the 6-hydroxydopamine insult was prevented by SA and FA administration. The degree of damage was higher in lesion group and L-dopa administrated group, this indicates that L-dopa does not prevent the degeneration of neurons whereas SA received animals showed a less degree of damage.

\section{DISCUSSION}

Our study demonstrates that the intrastriatal administration of 6-hydroxydopamine reduces the striatal neurotransmitters level, antioxidant activity in serum and shows the abnormal architecture of striatal and Midbrain tissues. This kind of effect proves that the 6-hydroxydopamine toxication is the ideal animal model for PD studies [25,26]. The levels of glucose, triglycerides, and protein were estimated, and we found significant alteration between the control, lesion and treated group. Previously, it was reported that immediately after L-dopa treatment the patients showed elevated level of hormones and plasma glucose and then it was reduced. At 1 year of chronic treatment of L-dopa, there was a decrease in glucose tolerance and associated with a delayed and exaggerated insulin response [27]. On the other hand, the SA and FA treatment have normalized these biological parameters indicating that they are safer to be consumed. Basically, phytochemicals are found to be safer than synthetically designed drugs. Many phytochemicals are used to treat chronic disease with no side effect [28-30]. This study suggests that SA is one among the safe phytochemicals that can be consumed. The study by Shooshtari et al. shows that the FA supplement has improved memory and motor activity of adult rats [31]. This supports our finding, as the SA and FA treated animals show normal level of FA in serum.

In our previous study, we have evaluated the behavioral activity of animals and antioxidant profile in the brain of experimental animals. We found that the animals induced with 6-hydroxydopamine showed abnormal behavioral activity and reduced antioxidant profile in the brain. On the other hand, there was an improvement in behavioral activity and antioxidant profile on SA and FA treatment [32]. Our study was in a harmony with other studies, such as quercetin showed neuroprotective effect against MPTP toxication in rats [33] and curcumin also showed neuroprotective effect against 6-hydroxydopamine toxicity [34]. As a well-known fact that the oxidative stress was found to be the main culprit for PD, it is important to evaluate the antioxidant profile of animals. In this study, the serum antioxidants were measured, and we found that the enzymatic antioxidant activities (SOD, catalase, GRx and GPx) and levels of GSH, vitamin-C and vitamin-E was reduced in the case of lesion group and it was restored by SA and FA treatment. On the other hand, the 6-hydroxydopamine induction increases the TBARS level in serum which in turn reduced on SA and FA treatment. The elevated level of TBARS indicates the occurrence of lipid peroxidation in the biological system. Lipid peroxidation is a complex reaction that involves the formation and propagation of lipid radical, uptake of oxygen, rearrangement in double bonds in unsaturated lipids and destruction in membrane lipids and producing a variety of breakdown products that includes alcohol, ketones, aldehyde and ether [35]. This lipid peroxidation chain reaction can be initiated by superoxide radical

Table 3: Non-enzymatic antioxidant activity in serum of experimental animals

\begin{tabular}{|c|c|c|c|c|c|}
\hline Antioxidants & Group 1 (control) & Group 2 (lesion) & Group 3 (lesion+SA) & Group 4 (lesion+SA+FA) & Group 5 (lesion+L-dopa) \\
\hline GSH ( $\mu \mathrm{g} / \mathrm{mg}$ protein) & $4.80 \pm 0.31$ & $2.49 \pm 0.41^{*}$ & $3.31 \pm 0.17^{\#}$ & $4.67 \pm 0.12^{\#}$ & $3.55 \pm 0.15^{\#}$ \\
\hline Vitamin-C (mg/dl) & $1.73 \pm 0.09$ & $0.98 \pm 0.09 *$ & $1.25 \pm 0.08^{\#}$ & $1.69 \pm 0.06^{\#}$ & $1.46 \pm 0.04^{\#}$ \\
\hline Vitamin-E (mg/dl) & $1.84 \pm 0.08$ & $1.02 \pm 0.07 *$ & $1.21 \pm 0.06^{\#}$ & $1.66 \pm 0.08^{\#}$ & $1.36 \pm 0.07^{\#}$ \\
\hline
\end{tabular}

The values were expressed as mean \pm SD, $n=6 .{ }^{*} p<0.001$ comparison between Group 1 and Group 2. " $p<0.001$, NS: Non-significant are comparison Group 3, Group 4 and

Group 5 with Group 2. SA: Sesamol, FA: Folic acid, GSH: Glutathione, SD: Standard deviation 

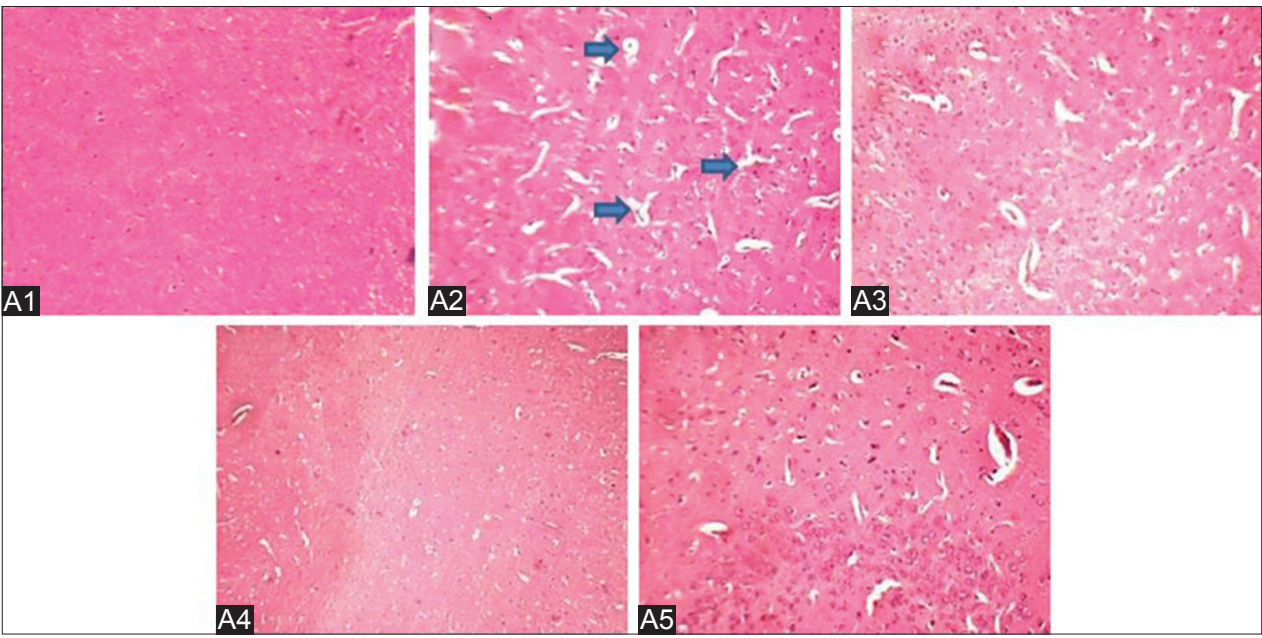

Plate A1-A5: Histopathology of striatal tissues $(\times 100)$. A1: Group 1 (control) possess a normal architecture, A2: Group 2 (lesion) the abnormalities like cellular inflammation, vascular degeneration and cytoplasmic vaculation are visualized (indicated by arrow), A3: Group 3 (lesion+SA), A4: Group 4 (lesion+SA+FA) showed a minimal degeneration and cytoplasmic vaculation, A5: Gropu 5 (lesion+Ldopa) similar as in Group 2 animal. SA: Sesamol, FA: Folic acid

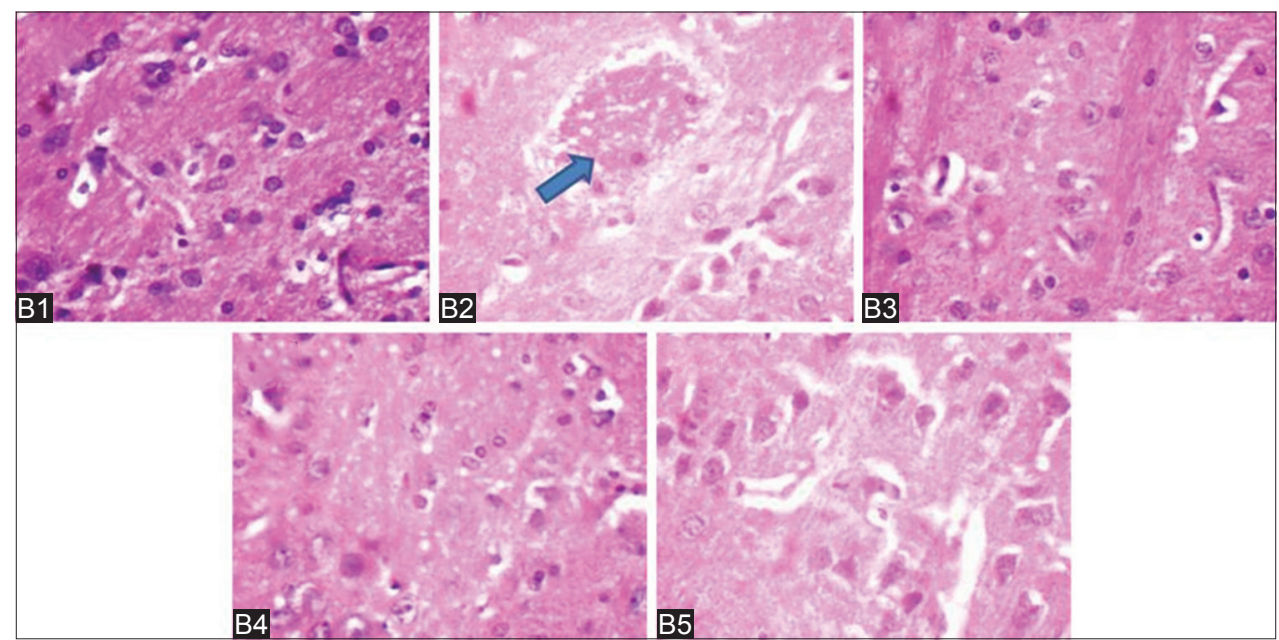

Plate B1-B5: Histopathology of mid-brain tissues (×100). B1: Group 1 (control) possess a normal architecture, B2: Group 2 (lesion) the abnormalities like cellular inflammation, vascular degeneration and cytoplasmic vaculation are visualized (indicated by arrow), B3: Group 3 (lesion+SA), B4: Group 4 (lesion+SA+FA) showed a minimal degeneration and cytoplasmic vaculation, B5: Group 5 (lesion+Ldopa) similar as in Group 2 animal. SA: Sesamol, FA: Folic acid

and nitric oxide [36]. The significant reduction in the TBARS level indicates the antioxidant scavenging activity of SA and FA.

The neurotransmitter levels in striatum of animals were depleted on 6-hydroxydopamine toxication which was restored on SA, FA, and L-dopa treatment. The reduced level of DA and its metabolite is the hallmark of PD. The DA is responsible for movement and thus its reduction leads to motor impairment. The NE, DOPAC, and HVA are the derivatives of DA. Hence, the reduction in the DA level also influences the level of these derivatives. It was reported that on intrastriatal administration of 6-hydroxydopamine specifically the dopaminergic neurons are degenerated by increased oxidative stress and apoptosis $[37,38]$. The degeneration of dopaminergic neurons will lead to depletion in the level of DA as dopaminergic neurons are the major source of DA. Restoration of the levels of these neurotransmitters was achieved by two ways either DA replacement therapy such as L-dopa supplement or by prevention or protection of dopaminergic neurons from degeneration. The prior one may cause many side effects, so the prevention of degeneration must be a better one. From the present investigation, SA in association with FA may prevent the degeneration of dopaminergic neurons and it was evidenced by restoration of the level of neurotransmitters.
The histopathological evidence shows that morphological changes like cellular inflammation, vascular degeneration and cytoplasmic vacuolation induced by 6-hydroxydopamine was restored on SA and FA treatment. SA and FA have a protective effect on striatal neurons and show a clear texture of striatal tissue as similar to the control group. On the other hand, the L-dopa could only alleviate the symptoms and it does not prevent degeneration or protect the neurons.

SA is supposed to stop the further degeneration of neurons induced by 6-hydroxydopamine and FA may act a supporting drug in preventing the degeneration from the day of administration. The neuronal number are found to be higher in the control group whereas the lesion animals showed only few neurons that are visible and this indicate that the neurons are degenerated due to 6-hydroxydopamine toxicity. A number of neurons are visualized more in SA and FA treated animals than lesion animals. This shows the neuroprotective effect of SA in association with FA. The present histopathological evidence was similar with other investigation which supports this study. The Lagenaria vulgaris showed neuroprotective effect against hypoxic neurotoxicity [39]. Similarly, valporic acid also showed a neuroprotective effect against 6-hydroxydopamine toxicity [40] 


\section{CONCLUSION}

The present investigation demonstrated that SA has nullified the toxicity of 6-hydroxydopamine. SA has reduced the oxidative stress, regularized antioxidant defense system, and increased the levels of neurotransmitters, prevented degeneration of dopaminergic neurons, which was evidenced by histopathological study of striatum and midbrain. In association with FA SA showed a better improvement. With these results, we emphasize that SA in association with FA shows better neuroprotective effect in treating Parkinsonian animals induced with 6-hydroxydopamine and it might serve as a novel therapeutic drug. Further investigations and clinical trials are required to justify the same.

\section{REFERENCES}

1. Wood-Kaczmar A, Gandhi S, Wood NW. Understanding the molecular causes of Parkinson's disease. Trends Mol Med 2006;12(11):521-8.

2. Dauer W, Przedborski S. Parkinson's disease: Mechanisms and models. Neuron 2003;39(6):889-909.

3. Gatto EM, Carreras MC, Pargament GA, Riobo NA, Reides C, Repetto M, et al. Neutrophil function, nitric oxide, and blood oxidative stress in Parkinson's disease. Mov Disord 1996;11(3):261-7.

4. Seidl SE, Potashkin JA. The promise of neuroprotective agents in Parkinson's disease. Front Neurol 2011;2:68.

5. Rangarajan N, Muthukumar V, Vanisree J. Neuroprotective effect of sesamol in glioma induced in rats. Biomed Int 2011;2:22-7.

6. Prasad NR, Mahesh T, Menon VP, Jeevanram RK, Pugalendi KV. Photoprotective effect of sesamol on UVB-radiation induced oxidative stress in human blood lymphocytes in vitro. Environ Toxicol Pharmacol $2005 ; 20(1): 1-5$.

7. Chandrasekaran VR, Hsu DZ, Liu MY. The protective effect of sesamol against mitochondrial oxidative stress and hepatic injury in acetaminophen-overdosed rats. Shock 2009;32(1):89-93.

8. Duan W, Ladenheim B, Cutler RG, Kruman II, Cadet JL, Mattson MP. Dietary folate deficiency and elevated homocysteine levels endanger dopaminergic neurons in models of Parkinson's disease. J Neurochem 2002;80(1):101-10.

9. Ziegler MG, Szechtman H. Relation between motor asymmetry and direction of rotational behaviour under amphetamine and apomorphine in rats with unilateral degeneration of the nigrostriatal dopamine system. Behav Brain Res 1990;39(2):123-33.

10. Ravindran R, Rathinasamy SD, Samson J, Senthilvelan M. Noisestress-induced brain neurotransmitter changes and the effect of Ocimum sanctum (Linn) treatment in albino rats. J Pharmacol Sci 2005;98(4):354-60.

11. Sasaki T, Matsy S, Sonae A. Effect of acetic acid concentration on the colour reaction in the o-toluidine boric acid method for blood glucose estimation. Rinsho Kagaku 1972;1:346-53.

12. Lowry OH, Rosebrough NJ, Farr AL, Randall RJ. Protein measurement with the Folin phenol reagent. J Biol Chem 1951;193(1):265-75.

13. Rice EW. Triglycerides ("neutral fats") in serum. In: Standard Methods of Clinical Chemistry. Vol. 6. New York: Academic Press, Inc.; 1970. p. 215-22.

14. Nagaraja P, Vasantha RA, Yathirajan HS. Spectrophotometric determination of folic acid in pharmaceutical preparations by coupling reactions with iminodibenzyl or 3-aminophenol or sodium molybdatepyrocatechol. Anal Biochem 2002;307(2):316-21

15. Marklund S, Marklund G. Involvement of the superoxide anion radical in the autoxidation of pyrogallol and a convenient assay for superoxide dismutase. Eur J Biochem 1974;47(3):469-74.

16. Sinha AK. Colorimetric assay of catalase. Anal Biochem 1972;47(2):389-94.

17. Rotruck JT, Pope AL, Ganther HE, Swanson AB, Hafeman DG, Hoekstra WG. Selenium: Biochemical role as a component of glutathione peroxidase. Science 1973;179(4073):588-90.

18. Mohandas J, Marshall JJ, Duggin GG, Horvath JS, Tiller DJ. Differential distribution of glutathione and glutathione related enzymes in rabbit kidneys: Possible implication in analgesic neuropathy. Cancer Res 1984:44:5086-91.

19. Moron MS, Depierre JW, Mannervik B. Levels of glutathione, glutathione reductase and glutathione S-transferase activities in rat lung and liver. Biochim Biophys Acta 1979;582(1):67-78.

20. Omaye ST, Turnbull JD, Sauberlich HE. Selected methods for the determination of ascorbic acid in animal cells, tissues, and fluids. Methods Enzymol 1979;62:3-11.

21. Desai ID. Vitamin E analysis methods for animal tissues. Methods Enzymol 1984;105:138-47.

22. Ohkawa H, Ohishi N, Yagi K. Assay for lipid peroxides in animal tissues by thiobarbituric acid reaction. Anal Biochem 1979;95(2):351-8.

23. Kari HP, Davidson PP, Kohl HH, Kochhar MM. Effects of ketamine on brain monoamine levels in rats. Res Commun Chem Pathol Pharmacol 1978;20(3):475-88.

24. Murphy GF, Robinson D, Sharman DF. The effect of tropolone on the formation of 3, 4-dihydroxyphenylacetic acid and 4-hydroxy-3methoxyphenylacetic acid in the brain of the mouse. $\mathrm{Br} \mathrm{J}$ Pharmacol 1969;36(1):107-15.

25. Courtière A, Hardouin J, Locatelli V, Turle-Lorenzo N, Amalric M, Vidal F, et al. Selective effects of partial striatal 6-OHDA lesions on information processing in the rat. Eur J Neurosci 2005;21(5):1973-83.

26. Simola N, Morelli M, Carta AR. The 6-hydroxydopamine model of Parkinson's disease. Neurotox Res 2007;11(3-4):151-67.

27. Sirtori CR, Bolme P, Azarnoff DL. Metabolic responses to acute and chronic L-dopa administration in patients with parkinsonism. N Engl J Med 1972;287(15):729-33

28. Palombo EA. Phytochemicals from traditional medicinal plants used in the treatment of diarrhoea: Modes of action and effects on intestinal function. Phytother Res 2006;20(9):717-24.

29. Kim J, Lee HJ, Lee KW. Naturally occurring phytochemicals for the prevention of Alzheimer's disease. J Neurochem 2010;112(6):1415-30.

30. Youdim KA, Joseph JA. A possible emerging role of phytochemicals in improving age-related neurological dysfunctions: A multiplicity of effects. Free Radic Biol Med 2001;30(6):583-94.

31. Shooshtari MK, Moazedi AA, Parham GA. Memory and motor coordination improvement by folic acid supplementation in healthy adult male rats. Iran J Basic Med Sci 2012;15(6):1173-9.

32. Khadira SA, Priya N, Vijayalakshmi K. Effect of sesamol and folic acid on behavioural activity and antioxidant profile of rats induced with 6-hydroxy dopamine. Int J Pharmacogn Phytochem Res 2015;6(4):930-93.

33. Pal A, Pany S, Sahu PK. Neuroprotective effect of quercetin in neurotoxicity induced rats: Role of neuroinflammation in neurodegeneration. Asian J Pharm Clin Res 2014;2:152-6.

34. Khuwaja G, Khan MM, Ishrat T, Ahmad A, Raza SS, Ashafaq M, et al. Neuroprotective effects of curcumin on 6-hydroxydopamineinduced Parkinsonism in rats: Behavioral, neurochemical and immunohistochemical studies. Brain Res 2011;1368: 254-63.

35. Buege JA, Aust SD. Microsomal lipid peroxidation. Methods Enzymol 1978;52:302-10.

36. Rafael R, Beckman JS, Bush KM, Freeman BA. Peroxynitrite-induced membrane lipid peroxidation: The cytotoxic potential of superoxide and nitric oxide. Arch Biochem Biophys 1991;288(2):481-7.

37. Zuch CL, Nordstroem VK, Briedrick LA, Hoernig GR, Granholm AC, Bickford PC. Time course of degenerative alterations in nigral dopaminergic neurons following a 6-hydroxydopamine lesion. J Comp Neurol 2000;427(3):440-54.

38. Deumens R, Blokland A, Prickaerts J. Modeling Parkinson's disease in rats: An evaluation of 6-OHDA lesions of the nigrostriatal pathway. Exp Neurol 2002;175(2):303-17.

39. Adnaik RS, Mohite SK. Neuroprotective evaluation of Lagenaria vulgaris extract hypoxic neurotoxicity induced rats. Asian J Pharm Clin Res 2015;8(4):121-4.

40. Machado XJ, Neves KR, Leal LK, Santos do Carmo MR, de Castro Brito GA, Naffah-Mazzacoratti MG, et al. Valproic acid neuroprotection in the 6-OHDA model of Parkinson's disease is possibly related to its anti-inflammatory and HDAC inhibitory properties. J Neurodegener Dis 2015;2015:1-14. 